\title{
Evaluating Treatment Response of Radioembolization in Intermediate-Stage Hepatocellular Carcinoma Patients Using ${ }^{18}$ F-Fluoroethylcholine PET/CT
}

\author{
Markus Hartenbach ${ }^{1}$, Stefan Weber ${ }^{1}$, Nathalie L. Albert ${ }^{2}$, Sabrina Hartenbach ${ }^{3}$, Albert Hirtl ${ }^{1}$, Mathias J. Zacherl ${ }^{2}$, \\ Philipp M. Paprottka ${ }^{4}$, Reinhold Tiling ${ }^{2}$, Peter Bartenstein ${ }^{2}$, Marcus Hacker ${ }^{1}$, and Alexander R. Haug ${ }^{1,2}$ \\ ${ }^{1}$ Division of Nuclear Medicine, Department of Biomedical Imaging and Image Guided Therapy, Medical University of Vienna, \\ Vienna, Austria; ${ }^{2}$ Department of Nuclear Medicine, Ludwig-Maximilians-University of Munich, Munich, Germany; ${ }^{3}$ Institute of \\ Pathology, German Armed Forces Hospital Ulm, Ulm, Germany; and ${ }^{4}$ Department of Clinical Radiology, Ludwig-Maximilians- \\ University of Munich, Munich, Germany
}

\begin{abstract}
The aim of this study was to evaluate ${ }^{18} \mathrm{~F}$-fluoroethylcholine PET/CT as a metabolic imaging technique for the assessment of treatment response to ${ }^{90} \mathrm{Y}$ radioembolization in patients with locally advanced hepatocellular carcinoma (HCC). Methods: Thirty-four HCC patients undergoing $78{ }^{18} \mathrm{~F}$-fluoroethylcholine PET/CT scans were identified for this study. Patients with initial or follow-up metastastic disease $(n=9)$ were excluded at the time point of the metastatic occurrence as well as patients with negative a-fetoprotein (AFP; $n=1$ ), resulting in 24 patients and 57 scans that were eligible. All patients were scheduled for radioembolization and underwent 1 pretherapeutic and at least 1 posttherapeutic ${ }^{18} \mathrm{~F}$-fluoroethylcholine PET/CT scan. Volume-of-interest analysis and volume-of-interest subtractions were performed. Maximum, mean, and peak standardized uptake value (SUV) analysis was performed, and the total intrahepatic ${ }^{18} \mathrm{~F}$-fluoroethylcholine positive tumor volume (FEC-PTV) and tumorto-background ratio were assessed. Statistical analysis was performed using a decreasing AFP of at least $20 \%$ as a standard of reference for therapy response including receiver-operatingcharacteristic analyses as well as descriptive and correlation analyses and multiple logistic regression. Results: Fourteen follow-up examinations were categorized as responder and 19 follow-up examinations as nonresponder. Absolute AFP values did not correlate with SUV parameters $(P=0.055)$. In receiver-operating-characteristic analyses, the initial mean SUV, $\Delta$ maximum SUV, and $\Delta$ tumor-tobackground ratio demonstrated the highest area under the curve, $0.84(P=0.009), 0.83(P=0.011)$, and $0.83(P=0.012)$, respectively, resulting in a positive prediction of $82 \%, 83 \%$, and $91 \%$ at the respective cutoff points. When multiple logistic regression analysis was applied, this resulted in an area under the curve of $0.90(P=0.001)$, with a positive prediction of $94 \%$ and a sensitivity of $94 \%$. The FECPTV did not reach significance in the presented dataset. Conclusion: ${ }^{18} \mathrm{~F}$-fluoroethylcholine PET/CT demonstrates a high potential for follow-up assessment in the context of radioembolization in patients with locally advanced, but nonmetastatic, HCC and initially elevated AFP, possibly enabling early therapy monitoring independent of morphology.
\end{abstract}

Received Apr. 20, 2015; revision accepted Aug. 17, 2015

For correspondence or reprints contact: Alexander Haug, Department of Molecular Imaging and Image Guided Therapy, Division of Nuclear Medicine, Medical University of Vienna, Währinger Gürtel 18-20, 1090 Vienna, Austria. E-mail: alexander.haug@meduniwien.ac.at

Published online Sep. 24, 2015.

COPYRIGHT (c) 2015 by the Society of Nuclear Medicine and Molecular Imaging, Inc.
Key Words: oncology; Gl; PET/CT; radionuclide; therapy; FEC; PET; hepatocellular carcinoma; radioembolization; therapy

J Nucl Med 2015; 56:1661-1666

DOI: 10.2967/jnumed.115.158758

D iagnostic methods for the detection and therapy monitoring of hepatocellular carcinoma (HCC), which represents the most increasing entity of cancer-related mortality worldwide (1), are limited (2-5). The main reason for the limitations is the constricted ability of CT or MR imaging for the assessment of viable tumor cells in a morphologic, often dramatically altered liver due to fibrosis, necrosis, and also therapy-related effects (6-8). This affects especially the assessment of therapy response. But still functional metabolic imaging is not a part of the European Association for the Study of the Liver (EASL) criteria (9) basically because the most commonly used PET tracer, ${ }^{18} \mathrm{~F}-\mathrm{FDG}$, is limited for the detection of higher differentiated HCC (10). In cirrhotic livers, present in $80 \%$ of patients with HCC, diagnosis is based solely on imaging and histology is performed only in ambiguous cases. Further, histology of HCC is also challenging (11). But especially in these patients, the initial diagnosis often comes at a late stage, thus resulting in advanced tumor stages (12).

Therapy monitoring refers to the (modified) Response Evaluation Criteria In Solid Tumors (RECIST/mRECIST), which themselves lack accuracy (13). Blood serum markers such as $\alpha$-fetoprotein (AFP) can be used as a surrogate for therapy response, for example, in postsurgery patients, and AFP is significantly correlated with tumor growth but only if it is initially positive (14). When used as follow-up marker, AFP itself lacks accuracy in metastatic disease for assessing local therapy response and, in general, if it is initially negative $(14,15)$.

Therefore, a noninvasive imaging method, which can early and reliably differentiate between therapy responders and nonresponders, is highly needed. This method might enable oncologists to adapt therapeutic schemes in time for an improved therapeutic outcome.

Radiolabeled choline, as an established tracer for PET, has demonstrated high sensitivities in the detection and staging of HCC $(10,16)$ as well as initial promising performance in therapy response assessment, but only in a small number of patients in 
a subgroup analysis (16). Choline is transported into the cells via choline transporters or diffusion and phosphorylated by the choline kinase (17). Radiolabeled phosphocholine is then trapped in the cells and not further metabolized. Therefore, it can be used as a suitable surrogate for cell membrane biosynthesis.

According to the Barcelona Clinic Liver Cancer (BCLC) classification, liver-directed therapies such as transarterial chemoembolization are indicated for patients with local multinodular disease (BCLC stage B) (12). In these patients, who represent approximately $20 \%$ of HCC patients with a median survival time of $20 \mathrm{mo}$, radioembolization is also of increasing significance (18). For the purpose of our study, an advantage of this subgroup with advanced but liver-confined disease is the possibility to evaluate treatment-induced quantitative metabolic alterations of the liver lesions by PET imaging and to compare them with blood serum tumor markers, such as the AFP, not being affected by metastatic disease. Therefore, the assessment of local therapy response and the potential of ${ }^{18} \mathrm{~F}$-fluoroethylcholine PET/CT can be evaluated more independently from RECIST or mRECIST.

These facts encouraged us in carrying out this study to evaluate preand posttherapeutic ${ }^{18} \mathrm{~F}$-fluoroethylcholine PET/CT scans of patients with known HCC being scheduled for radioembolization at our clinic. The aim was to assess the potential of this metabolic hybrid imaging method for monitoring therapy response.

\section{MATERIALS AND METHODS}

This retrospective study was approved by the local ethics committee of the University of Munich, and the requirement to obtain informed consent was waived. Thirty-four patients with known HCC according to the EASL criteria or histology were identified for this analysis. All patients were scheduled for radioembolization and received in total 78 pre- and posttherapeutic ${ }^{18} \mathrm{~F}$-fluoroethylcholine PET/CT scans. Nine patients presented initial or follow-up extrahepatic disease, not being categorized stage B anymore and therefore not being suitable for this analysis. One patient was AFP-negative on initial and follow-up PET/ CT scans. In total, 24 patients with 57 scans ( 24 before radioembolization and 33 after radioembolization) were eligible and analyzed. The interval for postradioembolization PET/CT examinations was $3 \mathrm{mo}$. Four patients underwent 3 PET/CT follow-up examinations, and 1 patient underwent 2 PET/CT scans after radioembolization. All additional follow-up PET/CT scans were compared with the initial preradioembolization PET/CT scans (Fig. 1).

Before radioembolization, all patients underwent angiography to evaluate vascular anatomy and identify relevant aberrant vessels. When necessary, prophylactic embolization of these vessels was performed (19). SIR-Spheres (SIRTeX Medical) were applied directly into the right and left hepatic arteries. The necessary activity was calculated using the body surface area (BSA) method (19): activity in gigabecquerel $(\mathrm{GBq})=(\mathrm{BSA}-0.2)+($ liver involvement $[\%] / 100)$.

Patients $(75 \% ; n=18)$ received dual-time-point radioembolization with a (median) 4-wk interval between right and left lobe therapy. Five patients had a single radioembolization of both lobes, and 1 patient received only segmental radioembolization in segments IVa $+\mathrm{b}$. The median injected doses were $1 \mathrm{GBq}( \pm 283 \mathrm{MBq})$ for the right lobe and $479 \mathrm{MBq}( \pm 195 \mathrm{MBq})$ for the left lobe. For single radioembolization, the median injected dose was $1,505 \mathrm{MBq}( \pm 443 \mathrm{MBq})$.

The posttherapeutic interval for ${ }^{18} \mathrm{~F}$-fluoroethylcholine PET/CT followup scans was $12 \pm 3 \mathrm{wk}$, starting after the second therapy in cases of dual-time-point radioembolization. Whole-body PET scans were acquired in 3-dimensional mode using one of two 64-slice PET/CT scanners (Biograph 64 TruePoint [Siemens Medical Solutions] or Discovery [GE Healthcare]). Before the CT scan, mean weight-adapted iodine- containing contrast agent (120 mL; Iopromide, Imeron 300; Bracco) was intravenously administered at a rate of $2.5 \mathrm{~mL} / \mathrm{s}$. Initiation of the CT scan (200-250 mA; $120 \mathrm{kV}$; collimation, $5 \times 5$ and $3 \times 3 \mathrm{~mm}$; pitch, 0.6) was delayed by $50 \mathrm{~s}$ after starting contrast agent infusion in order to depict the venous contrast medium phase. The PET emission scan was initiated $60 \mathrm{~min}$ after intravenous injection of ${ }^{18} \mathrm{~F}$-fluoroethylcholine ( $3 \mathrm{MBq} / \mathrm{kg}$ of body weight). Emission data were reconstructed with attenuation correction on the basis of concurrent diagnostic CT. FEC was produced and delivered by PETNet GmbH.

For image analysis, Siemens Syngo TrueD workstations were used. Detailed volume-of-interest (VOI) analysis and subtractions were performed using Hermes Hybrid 3D (Hermes Medical Solutions). Liver VOIs for total liver volume (TLV) were drawn manually on CT images using region-of-interest interpolation. Maximum, mean, and peak standardized uptake value $\left(\mathrm{SUV}_{\text {max }}, \mathrm{SUV}_{\text {mean }}\right.$, and $\mathrm{SUV}_{\text {peak }}$, respectively) analysis was performed with a 3-dimensional VOI and a $50 \%$ isocontour. The analysis of the intrahepatic ${ }^{18} \mathrm{~F}$-fluoroethylcholine positive tumor volume (FEC-PTV) was threshold-based. The lower threshold was defined as being $50 \%$ higher than the $\mathrm{SUV}_{\max }$ taken from a cubic VOI out of a non-HCC-affected area of the liver (defined by CT) (Fig. 2). As long as only venous-phase CT scans were obtained in this PET/CT approach, other multiphase CT or multiphase MR images obtained in

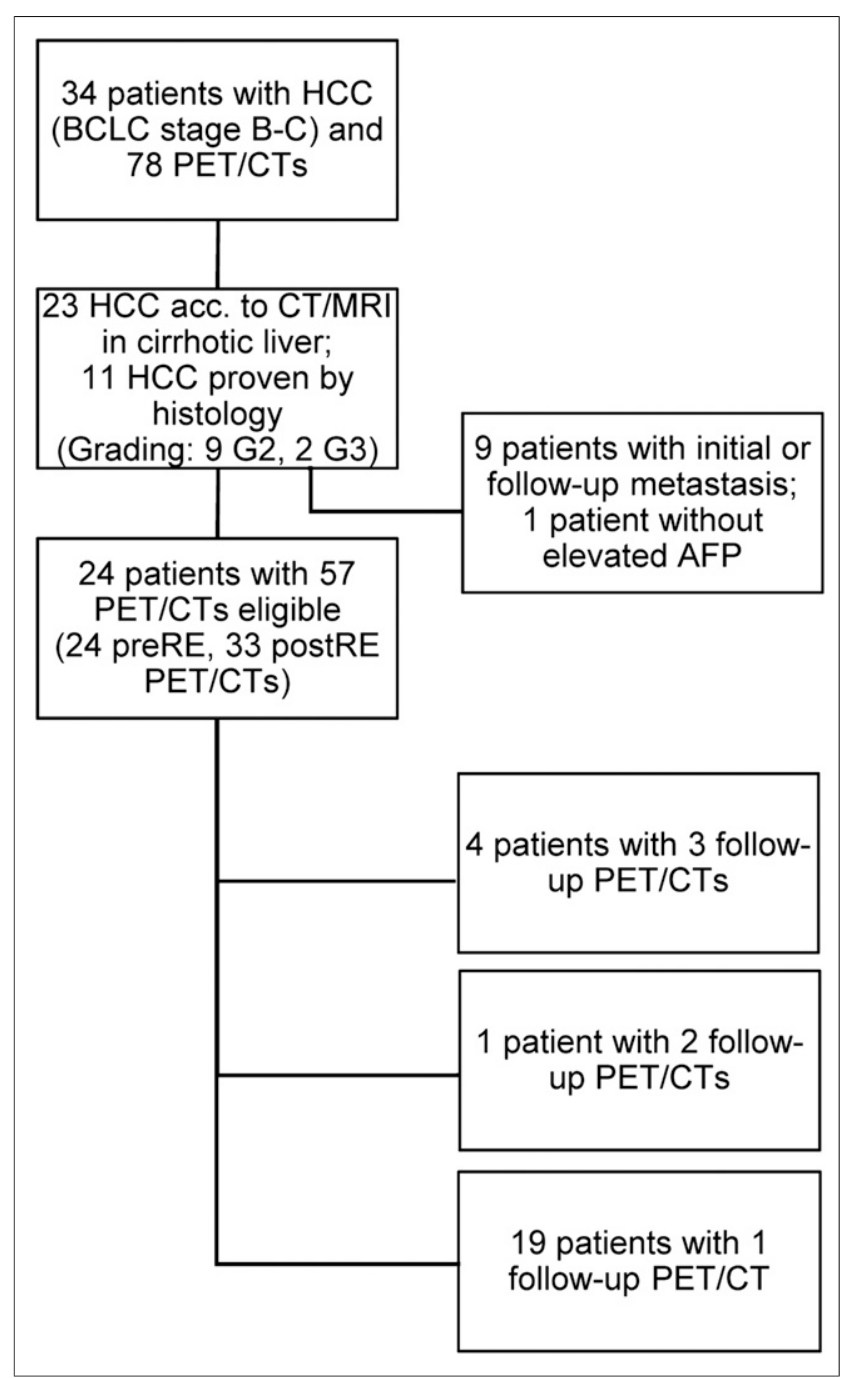

FIGURE 1. Patient accrual of study. acc. = according; $\mathrm{RE}=$ radioembolization. 
TABLE 1

Results of FEC SUV Analyses at ROC Cutoff

\begin{tabular}{|c|c|c|c|c|}
\hline Parameter & $\begin{array}{l}\text { Postradioembolization } \\
\text { SUV }_{\text {mean }} \text { cutoff, } 12.4\end{array}$ & $\begin{array}{l}\Delta \mathrm{SUV}_{\max } \\
\text { cutoff, }-3.5\end{array}$ & $\begin{array}{l}\Delta \text { SUV }_{\max } \text { TBR } \\
\text { cutoff, } 0.72\end{array}$ & $\begin{array}{l}\text { Multiple logistic } \\
\text { regression } \\
\text { cutoff, }-0.68\end{array}$ \\
\hline Sensitivity & $93 \%(77-100)$ & $100 \%(84-100)$ & $67 \%(48-73)$ & $94 \%(77-99)$ \\
\hline Specificity & $63 \%(31-74)$ & $63 \%(33-63)$ & $88 \%$ (53-99) & $88 \%$ (55-99) \\
\hline Accuracy & $83 \%(61-91)$ & $87 \%(66-87)$ & $74 \%(50-82)$ & $92 \%(70-99)$ \\
\hline $\begin{array}{l}\text { Positive predictive } \\
\text { value }\end{array}$ & $82 \%(68-88)$ & $83 \%(70-83)$ & $91 \%(66-100)$ & $94 \%(77-99)$ \\
\hline $\begin{array}{l}\text { Negative predictive } \\
\text { value }\end{array}$ & $83 \%(42-99)$ & $100 \%(52-100)$ & $58 \%(36-66)$ & $88 \%$ (55-99) \\
\hline AUC & 0.84 & 0.83 & 0.83 & 0.90 \\
\hline$P(\mathrm{AUC})$ & 0.009 & 0.011 & 0.012 & 0.001 \\
\hline
\end{tabular}

ROC cutoff values were derived by respective Youden indices. Values below cutoff indicate therapy response, defined as greater than $20 \%$ decrease of AFP. Multiple logistic regression represents combination of first 3 parameters by equation: $\operatorname{Logit}_{(\mathrm{P})}=2.96 \times \mathrm{SUV} \mathrm{mean}^{-}$ $0.95 \times \Delta$ SUV $_{\max }+0.19 \times \Delta$ SUV $_{\text {maxTBR. }}$. For TBR, background $=$ spleen. Data in parentheses are $95 \%$ confidence intervals.

each patient before radioembolization were considered to confirm these nonaffected areas. The relationship between the FEC-PTV and the TLV, measured on CT images and defined as volumetric ${ }^{18} \mathrm{~F}$-fluoroethylcholine tumor-to-liver ratio, was also determined according to the formula:

$$
\text { Volumetric FEC tumor-to-liver ratio }=\frac{\text { FEC-PTV }}{\text { TLV }}
$$

Additionally, tumor-to-background ratios (TBRs) were calculated. As far as the spleen is an organ with relatively high but homogeneous fluoroethylcholine uptake, we defined a cuboid VOI of the spleen (5-voxel side length) and calculated TBRs for $\mathrm{SUV}_{\text {max }}, \mathrm{SUV}_{\text {mean }}$, and $\mathrm{SUV}_{\text {peak }}$ according to the formula:

$$
\mathrm{TBR}=\frac{\text { SUV (tumor) }}{\text { SUV (spleen) }}
$$

where SUV is standardized uptake value. According to the $\triangle \mathrm{AFP}$ (AFP alteration between the PET/CT scans), the $\Delta$ of all PET/CT parameters was also determined, for example, the $\Delta$ of $\mathrm{SUV}_{\operatorname{maxTBR}}$ was calculated according to the formula:

$$
\Delta \mathrm{SUV}_{\max T B R}=\mathrm{SUV}_{\max \mathrm{TBR}} \text { postRE }-\mathrm{SUV}_{\max \mathrm{TBR}} \text { preRE},
$$

where RE is radioembolization. Statistical analysis was performed using SigmaPlot 12.0 (Systat Tech Inc.). Data derived from SUV measurements were analyzed for correlation with AFP using Pearson correlation. Descriptive analysis comprised the determination of the respective mean, maximum, minimum, and median values. Patients were defined as therapy responder when AFP decreased more than $20 \%$ from preradioembolization to postradioembolization PET/CT or in between the postradioembolization PET/CT scans and vice versa for being described as a therapy nonresponder. This dichotomic parameter was used for receiver-operating-characteristic (ROC) analyses and descriptive and correlation analyses with SUVs. Cutoff values derived from ROC analyses and the respective Youden indices served as a basis for calculating contingency tables as well as sensitivity, specificity, and negative and positive prediction values. $95 \%$ confidence intervals were calculated. For evaluating a synergistic use of the derived PET parameters, multiple logistic regression analysis of the SUV parameters with the highest area under the curve (AUC) was performed. ROC curve area comparison was performed, and the respective level of significance was determined using the Delong und Clarke-Pearson method for paired groups.

\section{RESULTS}

Of the 34 patients undergoing pre- and posttherapeutic ${ }^{18} \mathrm{~F}$-fluoroethylcholine PET/CT, 11 had HCC proven by histology and 23 were diagnosed according to the EASL criteria. After exclusion of the 10 patients with metastases of negative AFP, 24 patients with 33 followup PET/CT scans were analyzed. Nineteen follow-up scans in patients who presented an increasing AFP were categorized as nonresponse. Fourteen follow-up scans with decreasing AFP were categorized as therapy response (Fig. 3). Two of the patients with an additional 2 PET/CT scans had an increasing AFP-1 patient had an initially decreasing and then again increasing AFP (one turned into reference ranges and remained normal), and the patient with 1 additional PET/ CT scan had a decreased AFP on both scans. Absolute AFP values did not correlate with SUV parameters in Pearson correlation analysis $(P=0.055)$. When ROC analyses were applied, the initial pretherapeutic $\mathrm{SUV}_{\text {mean }}, \Delta \mathrm{SUV}_{\text {max }}$ (pre- to posttherapeutic), and $\triangle \mathrm{SUV}_{\text {maxTBR }}$ (pre- to posttherapeutic) demonstrated the highest AUC (Fig. 4), with $0.84(P=0.009), 0.83(P=0.011)$, and 0.83 $(P=0.012)$, respectively, resulting in a positive prediction of therapy nonresponders of $82 \%(P=0.009), 83 \%(P=0.002)$, and $91 \%(P=$ $0.002)$ at the respective cutoff points. Results are presented in Table 1.

The multiple logistic regression analysis of these data resulted in the equation:

$$
\begin{aligned}
\operatorname{Logit}(\mathrm{P})= & 2.96 \times \mathrm{SUV}_{\text {mean }}-0.95 \times \Delta \mathrm{SUV}_{\text {max }} \\
& +0.19 \times \Delta \mathrm{SUV}_{\text {maxTBR }}
\end{aligned}
$$

Applying this equation to the present dataset resulted in an AUC of $0.90(P=0.001)$, with an improved positive prediction of $94 \%$ and an also increased sensitivity of $94 \%(P<0.001$; Fig. 4; Table 1). However, when comparing the AUC of $\mathrm{SUV}_{\text {mean }}$ with the AUC of $\operatorname{Logit}(\mathrm{P})$, the level of significance was slightly not reached $(P=0.073)$. 


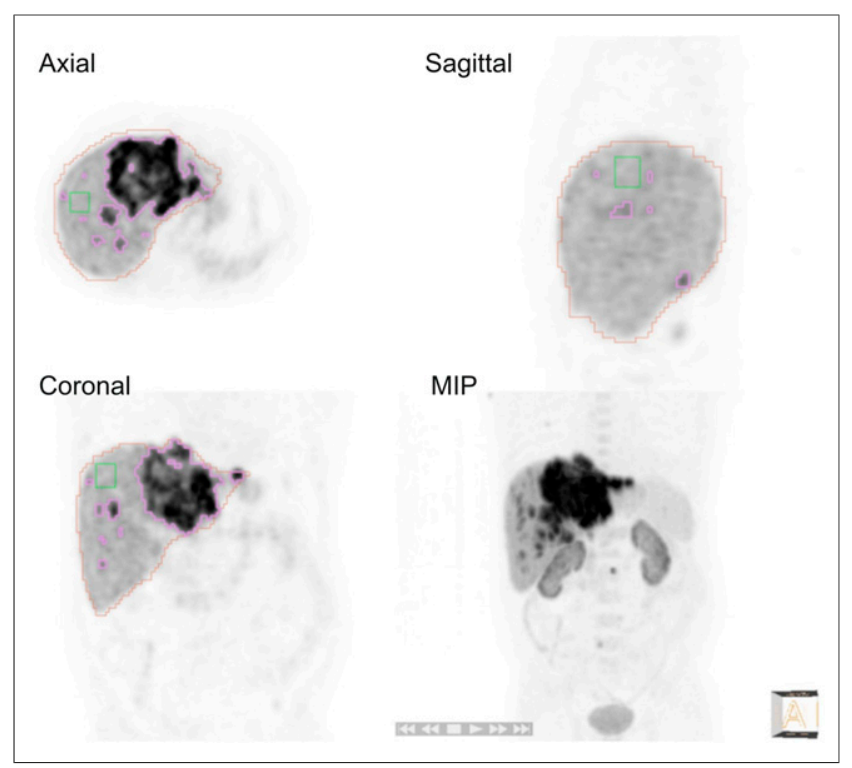

FIGURE 2. VOI analyses of intrahepatic FEC-PTV (50\% above background $\left.S U V_{\text {max }}\right)$. Background was determined via defined cubic VOI (green). Purple VOI demonstrates threshold-based FEC tumor VOI. Orange VOI was drawn manually on CT images and copied to PET image to determine TLV. MIP = maximum-intensity projection

The $\mathrm{SUV}_{\max }$ tumor-to-liver ratio in our cohort was $3.21 \pm 1.62$ (mean $\pm \mathrm{SD}$ ), with a minimum ratio of 1.67 (background $\mathrm{SUV}_{\max }$, $9.24 \pm 2.82)$. Nevertheless, the analysis of the volumetric data including the FEC-PTV as well as the volumetric ${ }^{18} \mathrm{~F}$-fluoroethylcholine tumor-to-liver ratio did not reach significance in the presented dataset. The highest AUC was reached by the $\triangle$ FEC-PTV and the $\triangle$ FEC-toliver-volume ratio (both AUC, $0.68 ; P=0.175 / 0.164$; Fig. 5).

\section{DISCUSSION}

Therapy monitoring using hybrid imaging is, particularly in complex and systemic diseases, a preferable method, as this may

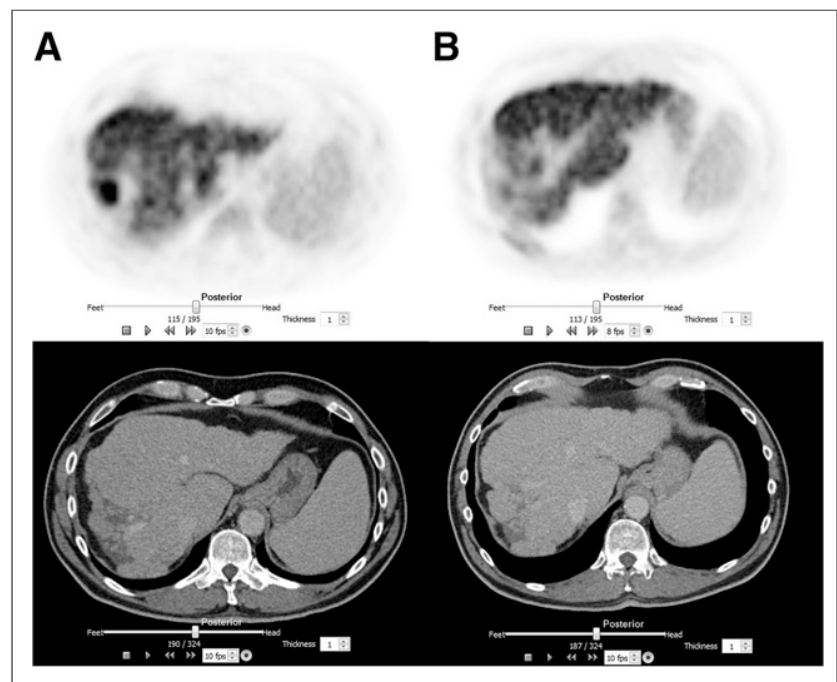

FIGURE 3. Metabolic response. Example of patient with progressive cirrhosis and severely altered morphology, especially in tumor-bearing area (segment VII). ${ }^{18} \mathrm{~F}$-fluoroethylcholine PET demonstrates significant metabolic response to radioembolization (before therapy $[\mathrm{A}]$; after therapy $[\mathrm{B}])$. AFP decreased by $50 \%$ into reference range. lead to a complete whole-body status including both morphologic and metabolic information. In HCC, however, hybrid imaging results in several challenges for both methods $(4-7,10)$. Assessing an early response to therapy after local or systemic treatment of HCC, however, according to morphologic aspects is limited (13); thus, the focus of our study was on the significance of the metabolic component of hybrid imaging using ${ }^{18} \mathrm{~F}$-fluoroethylcholine $\mathrm{PET} / \mathrm{CT}$.

All consecutive patients with HCC who were scheduled for radioembolization were considered in this study. To generate a homogeneous patient collective and to have a reference parameter for treatment response independent from image morphology, patients with extrahepatic metastases were not included in the analysis. This allowed the use of AFP as outcome measures but required the exclusion of initially AFP-negative patients, because AFP showed high potential in treatment monitoring in earlier studies only insofar as it was initially positive $(14,15)$. Although there is evidence in the literature using an AFP cutoff of $50 \%$ decrease for therapy responders with a baseline AFP $200 \mathrm{ng} / \mathrm{mL}$ (20), these studies starting with a large patient population had a large drop-out rate due to these criteria. In our cohort of patients in which an unestablished imaging method was applied, only 2 patients would have been regarded as therapy responders with these criteria applied. Therefore, we chose the AFP cutoff of a greater than $20 \%$ decrease for therapy responders, which is also discussed in the literature (20). Thus, we were able to evaluate ${ }^{18} \mathrm{~F}$-fluoroethylcholine PET/CT for the assessment of intrahepatic $\mathrm{HCC}$ after radioembolization.

Our study revealed that a TBR, using the spleen as an organ with a relatively high choline uptake as background, in connection with the initial $\mathrm{SUV}_{\text {mean }}$ indicated response to treatment with high accuracy. Although the total number of cases in this pilot study is low and the predictive power is therefore limited, we determined the positive and negative predictive values of the respective semiquantitative methods because our collective with $38 \%$ responders after radioembolization has a representative distribution according to the literature (21). With a $94 \%$ positive prediction, the regression analysis showed high potential to evaluate a treatment failure, which could have a direct impact on an early therapy adaptation. Therefore, functional imaging using ${ }^{18} \mathrm{~F}$-fluoroethylcholine PET/ CT seems to be able to evaluate early local tumor alterations and might therefore be a reliable parameter for response assessment in those HCC patients in whom AFP cannot be used for treatment monitoring - that is, those with metastases or initially negative AFP. This assumption needs to be evaluated, but our initial results can provide the basis for further clinical studies (e.g., in patients with sorafenib therapy), even in advanced HCC, in order to reveal the importance of ${ }^{18} \mathrm{~F}$-fluoroethylcholine $\mathrm{PET} / \mathrm{CT}$ in the therapeutic management of HCC.

In contrast to the SUVs, the analysis performed on the FECPTV and the volumetric FEC tumor-to-liver ratio yielded no significant results in the assessment of the course of the disease. On the one hand, this might be due to the applied image analysis method, as we used a threshold of $50 \%$ increased FEC uptake over a defined background VOI in noncancerous liver tissue, which might also involve inflammatory and cirrhotic inhomogeneous areas in the volume calculation (10). On the other hand, the overall metabolic volume might remain largely unchanged after radioembolization while the maximum choline-metabolizing areas increase or decrease and therefore appear to be more responsible for the course of the disease. A further analysis of this inhomogeneity 


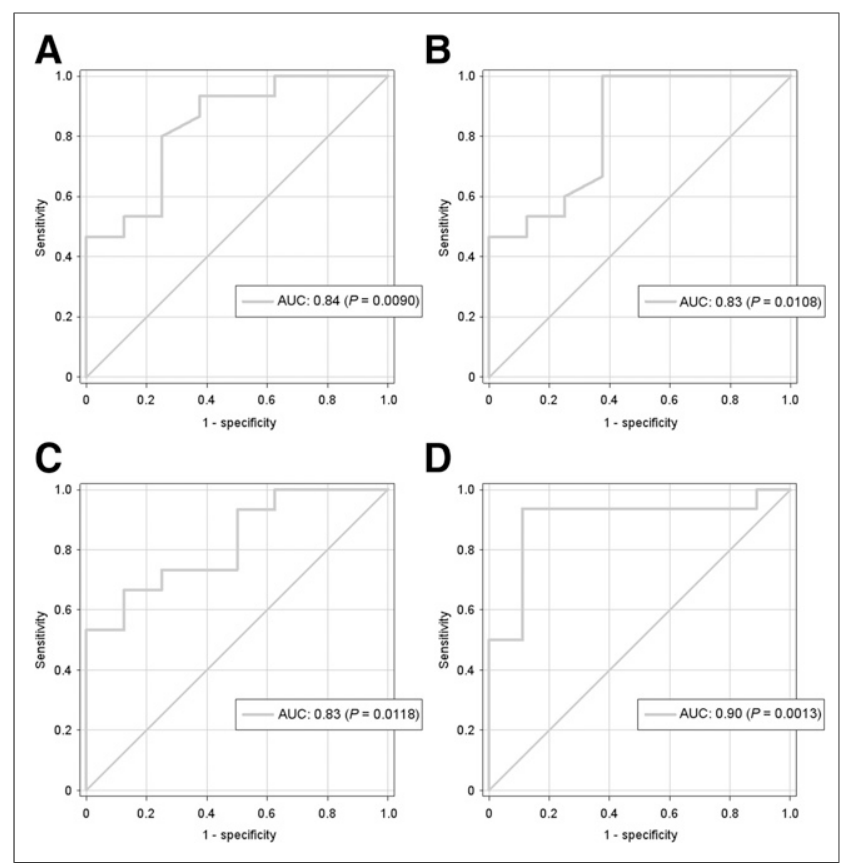

FIGURE 4. ROC analyses of SUVs and its alterations. ROC analysis of intrahepatic FEC tumor uptake. Semiquantitative parameters (postradioembolization) $S U V_{\text {mean }}(\mathrm{A}), \Delta \mathrm{SUV}_{\max }(\mathrm{B})$, and $\Delta S U V_{\text {maxtBR }}(\mathrm{C})$ demonstrated highest $A \cup C$ and were combined via multiple logistic regression (D). acc. = accuracy.

in the primary HCC lesion, with regard to both the metabolism and vascularization and the additional parameters of the MR imaging such as diffusion and perfusion, is the subject of current studies in our group. In the course of functional hybrid imaging using PET/MR imaging, these processes can be measured simultaneously in 1 examination and promise to improve the outcome of these patients significantly by early, noninvasive treatment monitoring.

Some limitations of our study beyond the AFP cutoff have to be mentioned. First, only patients without metastases were included in order to ensure the comparison with a reliable, not-imaging-based follow-up marker; therefore, additional studies are needed to assess the value of ${ }^{18} \mathrm{~F}$-fluoroethylcholine PET/CT in those patients, as discussed above. Although the patient cohort has a representative responder-to-nonresponder distribution, the total number of res- ponders is low and therefore limits the ability of comparing the sensitivities of the respective semiquantitative methods. Another limitation in the subanalysis of FEC-PTV is the choice of the HCC-nonaffected liver background VOI. Although it was confirmed by multiphase CT or MR imaging, a HCC involvement not detected by these methods might have been possible and could have affected this analysis, which was not significant in our patient cohort. This analysis might also be limited in smaller tumors with lower uptake as far as only areas with ${ }^{18} \mathrm{~F}$-fluoroethylcholine uptake $50 \%$ higher than the background $\mathrm{SUV}_{\max }$ were considered. In our patient cohort with locally advanced tumor stages and a mean tumor-to-liver ratio of $3.21 \pm 1.62$, this method did not produce drop-outs. On the other hand, applying automated methods of drawing VOIs based on background thresholds, rather than manually drawn VOIs, enables reproducibility. However, as our data demonstrate that the alterations of tumor areas with the highest FEC uptake (applying a 50\% isocontour of the $\mathrm{SUV}_{\max }$ ) are reliable surrogates for therapy response, the coverage of the exact extent of advanced HCCs might be of inferior value for therapy monitoring. Additionally, our results on metabolic response are not compared with RECIST or mRECIST. The main reason for that limitation is the acquisition of only venous contrast-enhanced CT data in the applied PET/CT protocol and only limited availability of follow-up multiphase CT or MR imaging investigations, which were acquired in an acceptable time span close to the PET/CT follow-ups. However, this study aimed at evaluating the accuracy of metabolic imaging and not the comparison between 2 imaging modalities. Nevertheless, prospective studies assessing radiographic and metabolic response criteria simultaneously are highly demanded and should be performed, for example, using dedicated PET/MR imaging scanners.

In addition, this type of metabolic hybrid imaging harbors the advantage of a more reliable whole-body staging than CT or MR imaging alone, so distant metastases can be detected earlier, enabling an early multimodal therapeutic approach. ${ }^{18} \mathrm{~F}$-fluoroethylcholine PET/CT, however, did already demonstrate promising results in the detection of metastatic disease of HCC $(10,16)$.

\section{CONCLUSION}

In the selective group of patients with locally advanced, but nonmetastatic, HCC and initially elevated AFP, ${ }^{18}$ F-fluoroethylcholine PET/CT demonstrated a high accuracy for response assessment in the context of radioembolization. This result might allow for early monitoring of local therapies independently from morphologic imaging and even in patients in whom serum tumor markers cannot be used for local treatment evaluation (e.g., in the case of metastatic disease or initially negative AFP). To confirm our findings, prospective randomized studies with multiparametric functional hybrid imaging as a basis for decisions are needed.

\section{DISCLOSURE}

The costs of publication of this article were defrayed in part by the payment of page charges. Therefore, and solely to indicate this fact, this article is hereby marked "advertisement" in accordance with 18 USC section 1734. No potential conflict of interest relevant to this article was reported.
FIGURE 5. ROC analyses of volumetric parameters and its alterations. Results were not significant. $\Delta$ of intrahepatic FEC-PTV (B and C) $\left(50 \%\right.$ above background SUV $\left.V_{\max }\right)$ is defined as: $\Delta$ FEC-PTV $=$ FEC-PTV postradioembolization - FEC-PTV preradioembolization. $\Delta$ of the volumetric FEC tumor-to-liver ratio $(\mathrm{A})$ is defined as: $\Delta$ volumetric $\mathrm{FEC}$ tumor-to-liver ratio $=(\mathrm{FEC}-$ PTV)/TLV postradioembolization - (FEC-PTV)/TLV preradioembolization. 


\section{REFERENCES}

1. Bosetti C, Levi F, Boffetta P, Lucchini F, Negri E, La Vecchia C. Trends in mortality from hepatocellular carcinoma in Europe, 1980-2004. Hepatology. 2008;48:137-145.

2. Stigliano R, Marelli L, Yu D, Davies N, Patch D, Burroughs AK. Seeding following percutaneous diagnostic and therapeutic approaches for hepatocellular carcinoma: what is the risk and the outcome? Seeding risk for percutaneous approach of HCC. Cancer Treat Rev. 2007;33:437-447.

3. Bruix J, Sherman M. Management of hepatocellular carcinoma: an update. Hepatology. 2011;53:1020-1022.

4. Forner A, Vilana R, Ayuso C, et al. Diagnosis of hepatic nodules $20 \mathrm{~mm}$ or smaller in cirrhosis: prospective validation of the noninvasive diagnostic criteria for hepatocellular carcinoma. Hepatology. 2008;47:97-104.

5. Bolondi L, Gaiani S, Celli N, et al. Characterization of small nodules in cirrhosis by assessment of vascularity: the problem of hypovascular hepatocellular carcinoma. Hepatology. 2005;42:27-34.

6. Colli A, Fraquelli M, Casazza G, et al. Accuracy of ultrasonography, spiral CT, magnetic resonance, and alpha-fetoprotein in diagnosing hepatocellular carcinoma: a systematic review. Am J Gastroenterol. 2006;101:513-523.

7. Jiang T, Zhu AX, Sahani DV. Established and novel imaging biomarkers for assessing response to therapy in hepatocellular carcinoma. J Hepatol. 2013;58: 169-177.

8. Forner A, Ayuso C, Varela M, et al. Evaluation of tumor response after locoregional therapies in hepatocellular carcinoma: are response evaluation criteria in solid tumors reliable? Cancer. 2009;115:616-623.

9. European Association for the Study of the Liver, European Organisation for Research and Treatment of Cancer. EASL-EORTC clinical practice guidelines: management of hepatocellular carcinoma. J Hepatol. 2012;56: 908-943.

10. Talbot JN, Fartoux L, Balogova S, et al. Detection of hepatocellular carcinoma with PET/CT: a prospective comparison of ${ }^{18} \mathrm{~F}$-fluorocholine and ${ }^{18} \mathrm{~F}$-FDG in patients with cirrhosis or chronic liver disease. J Nucl Med. 2010;51:1699-1706.
11. Roskams T, Kojiro M. Pathology of early hepatocellular carcinoma: conventional and molecular diagnosis. Semin Liver Dis. 2010;30:17-25.

12. Llovet JM, Bru C, Bruix J. Prognosis of hepatocellular carcinoma: the BCLC staging classification. Semin Liver Dis. 1999;19:329-338.

13. Edeline J, Boucher E, Rolland Y, et al. Comparison of tumor response by response evaluation criteria in solid tumors (RECIST) and modified RECIST in patients treated with sorafenib for hepatocellular carcinoma. Cancer. 2012; 118:147-156.

14. Yamamoto K, Imamura H, Matsuyama Y, et al. Significance of alpha-fetoprotein and des-gamma-carboxy prothrombin in patients with hepatocellular carcinoma undergoing hepatectomy. Ann Surg Oncol. 2009;16:2795-2804.

15. Vora SR, Zheng H, Stadler ZK, Fuchs CS, Zhu AX. Serum alpha-fetoprotein response as a surrogate for clinical outcome in patients receiving systemic therapy for advanced hepatocellular carcinoma. Oncologist. 2009;14:717-725.

16. Bieze M, Klumpen HJ, Verheij J, et al. Diagnostic accuracy of ${ }^{18} \mathrm{~F}$-methylcholine positron emission tomography/computed tomography for intra- and extrahepatic hepatocellular carcinoma. Hepatology. 2014;59:996-1006.

17. DeGrado TR, Baldwin SW, Wang S, et al. Synthesis and evaluation of ${ }^{18}$ F-labeled choline analogs as oncologic PET tracers. J Nucl Med. 2001; 42:1805-1814.

18. Salem R, Lewandowski RJ, Mulcahy MF, et al. Radioembolization for hepatocellular carcinoma using yttrium-90 microspheres: a comprehensive report of long-term outcomes. Gastroenterology. 2010;138:52-64.

19. Kennedy A, Nag S, Salem R, et al. Recommendations for radioembolization of hepatic malignancies using yttrium-90 microsphere brachytherapy: a consensus panel report from the radioembolization brachytherapy oncology consortium. Int J Radiat Oncol Biol Phys. 2007;68:13-23.

20. Riaz A, Ryu RK, Kulik LM, et al. Alpha-fetoprotein response after locoregional therapy for hepatocellular carcinoma: oncologic marker of radiologic response, progression, and survival. J Clin Oncol. 2009;27:5734-5742.

21. Vente MA, Wondergem M, van der Tweel I, et al. Yttrium-90 microsphere radioembolization for the treatment of liver malignancies: a structured meta-analysis. Eur Radiol. 2009;19:951-959. 